\title{
Poly-Methacrylic Acid Cross-linked with Collagen Accelerates Diabetic Wound Closure
}

\section{Supporting information}

Virginie F. Coindre ${ }^{1}$, Yangshuo $\mathrm{Hu}^{2}$, Michael V. Sefton ${ }^{1,2 *}$

${ }^{1}$ Institute of Biomedical Engineering, University of Toronto

${ }^{2}$ Department of Chemical Engineering and Applied Chemistry, University of Toronto.

*Corresponding author:

Michael V. Sefton

Institute of Biomedical Engineering

University of Toronto

164 College Street, Suite 407, Toronto, Ontario, Canada M5S 3G9

E-mail: michael.sefton@utoronto.ca

This document contains 7 supplementary figures. 

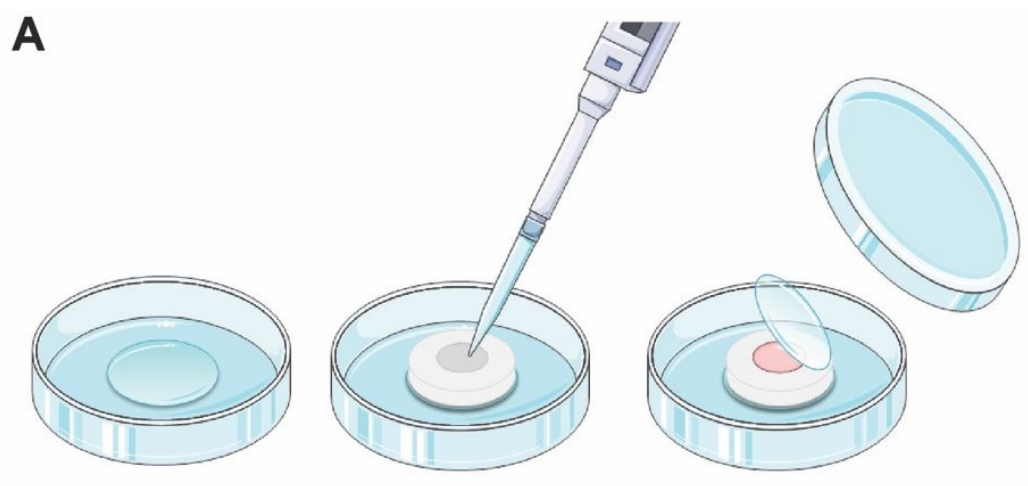

B

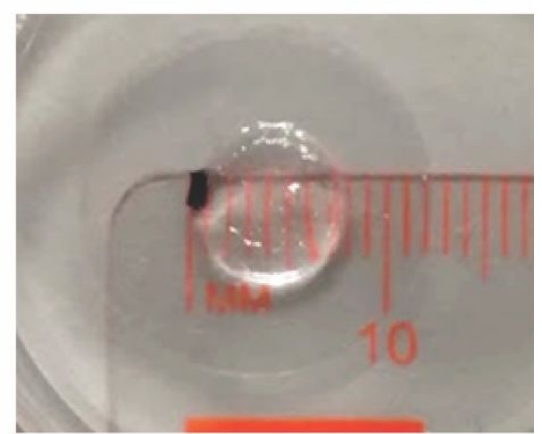

Figure S1: Fabrication of gel disks. (A) Collagen and polyMAA-collagen solutions were cast into a silicone mold, made from a silicone sheet with a $8 \mathrm{~mm}$ hole and placed between two coverslips. The resulting gel was $1 \mathrm{~mm}$ thick and the same $8 \mathrm{~mm}$ diameter of the wounds. (B) 8 mm diameter polyMAA-collagen disk.
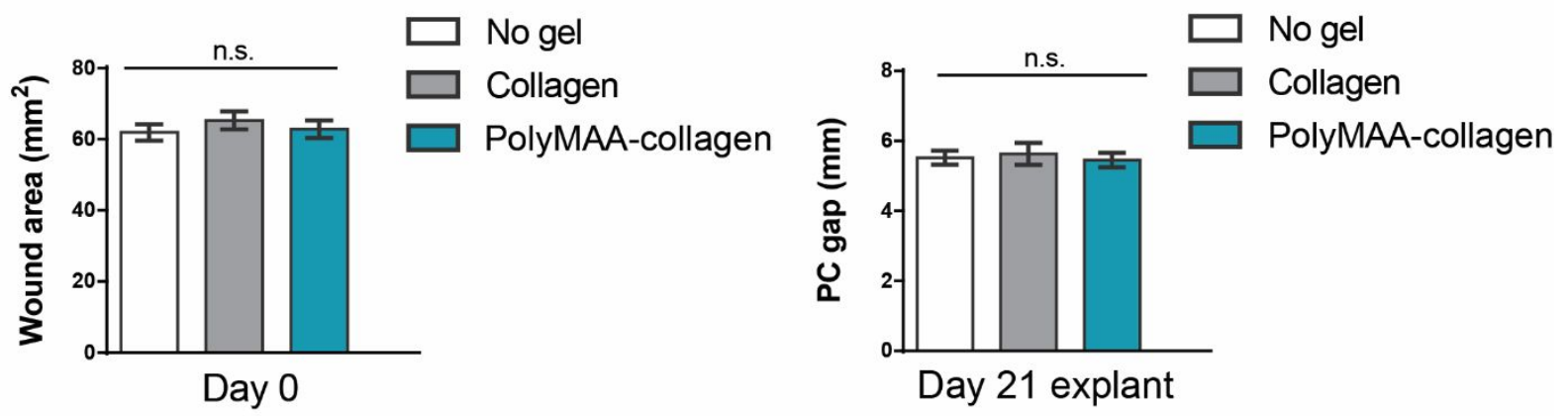

Figure S2: Animals had similar wound size among groups. (A) Wound area assessed by planimetry. (B) Panniculus carnosus gap at day 21 determined by histology, indicating that wound diameters were not different among groups. 
Day 0

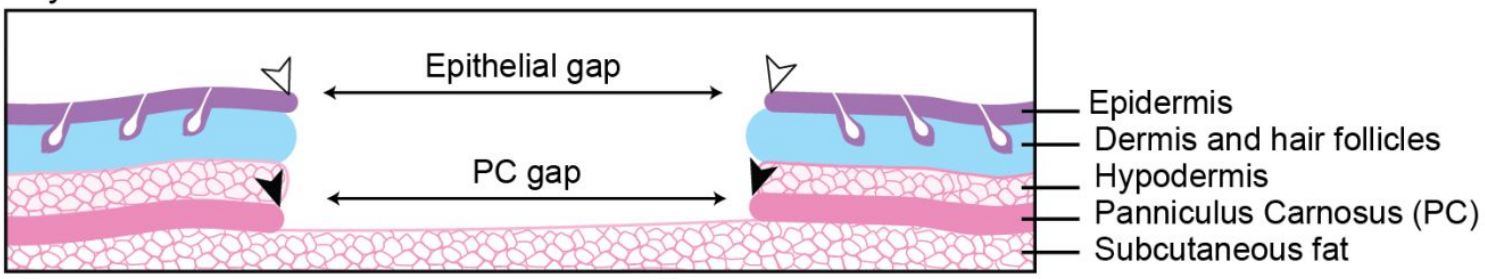

Few days after wounding

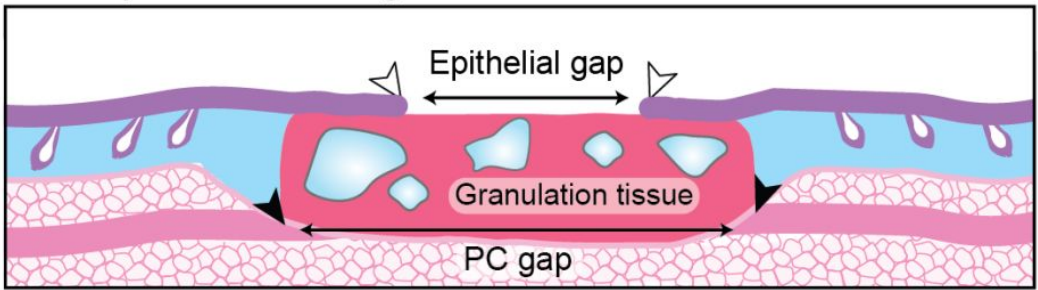

Figure S3: Schematic illustration of wound closure by re-epithelialization. The percent reepithelialization was defined as the ratio between the epithelial gap and the original wound. Granulation tissue region was defined as the area between the edges of panniculus carnosus (PC), the subcutaneous fat layer and the epidermis, excluding the residual gel.

A

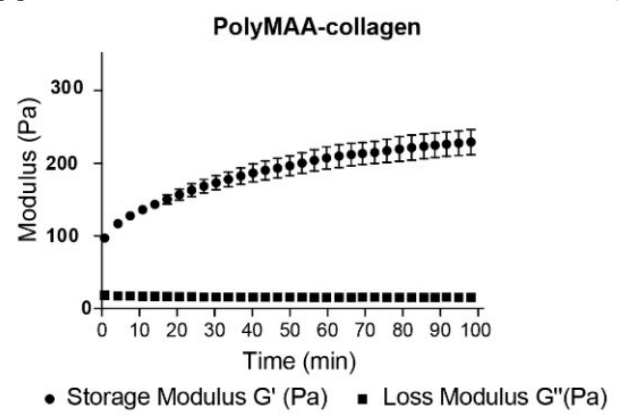

B

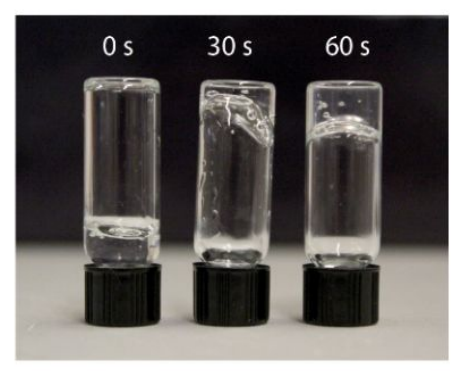

C

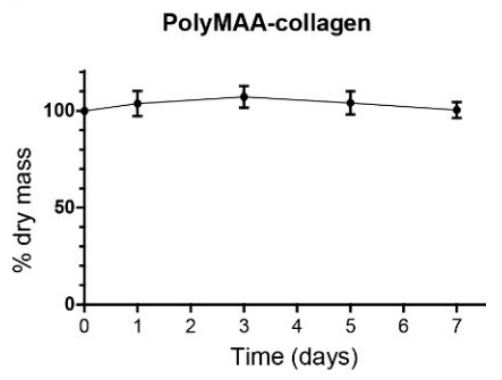

Figure S4: polyMAA-collagen gel had a storage modulus of $240 \mathrm{~Pa}$ and did not degrade in PBS. (A) Time sweep of polyMAA-collagen gel after gel mixture neutralization and the start of gelation. G' Storage modulus, G' Loss Modulus. (B) Gelation sufficient to hold shape under gravity occurred within 60 seconds after the $\mathrm{pH}$ was neutralized. (C) PolyMAA-collagen gel's dry mass was constant over time of incubation in PBS. Data presented as mean $\pm \mathrm{SE}$. 
A $\square$ No gel $\square$ Collagen $\square$ PolyMAA-collagen
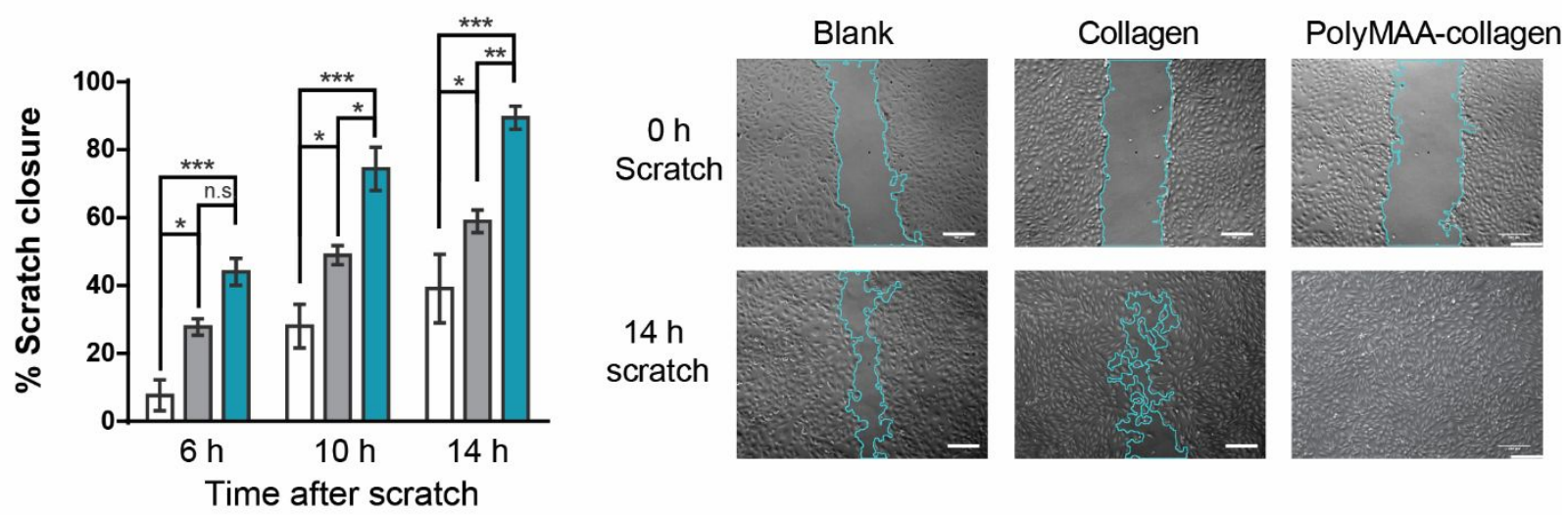

$14 \mathrm{~h}$

scratch
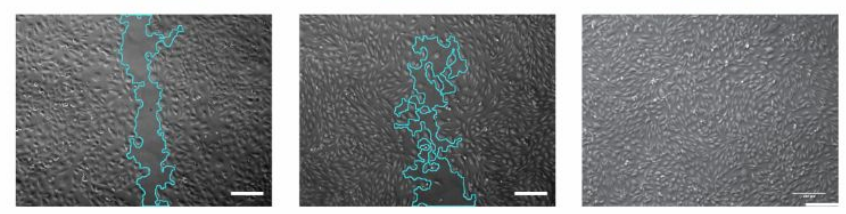

Figure S5: Endothelial cells incubated with conditioned media from dTHP1 cells previously cultured on polyMAA-collagen gel migrated further in a scratch wound assay. (A) PolyMAAcollagen conditioned medium increased the migration (scratch closure) of HUVEC that had been scratched compared to groups with collagen gel, or no gel. (B) Representative brightfield images of HUVEC before and after 14 hours of incubation with conditioned media. Data are presented as mean \pm SE. Scale bar $=200 \mu \mathrm{m} .{ }^{*} \mathrm{p}<0.05,{ }^{*} \mathrm{p}<0.01,{ }^{* * *} \mathrm{p}<0.001$, n.s. not significant.

A Collagen gel remodeling

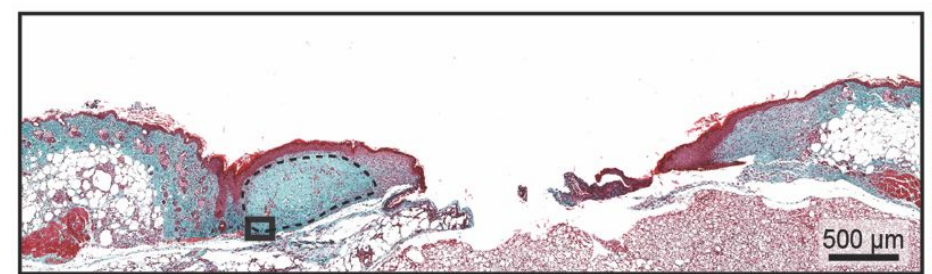

Figure S6: Collagen gel (no polyMAA) at day 21, that did not stain for CD68. (A) Representative histological pictures of the wounds in the collagen group stained with Masson trichrome or CD68 at day 21. (B-C) CD68 negative cells were present in the collagen gel at day 21.
B

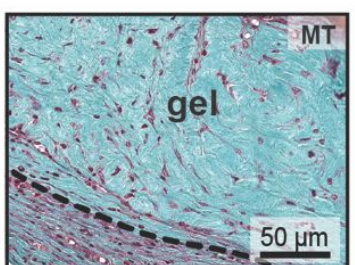

C Macrophages

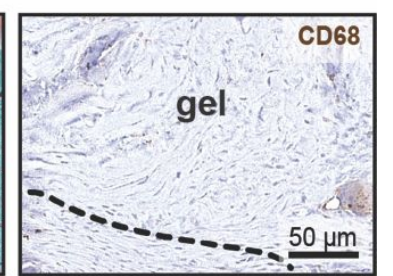




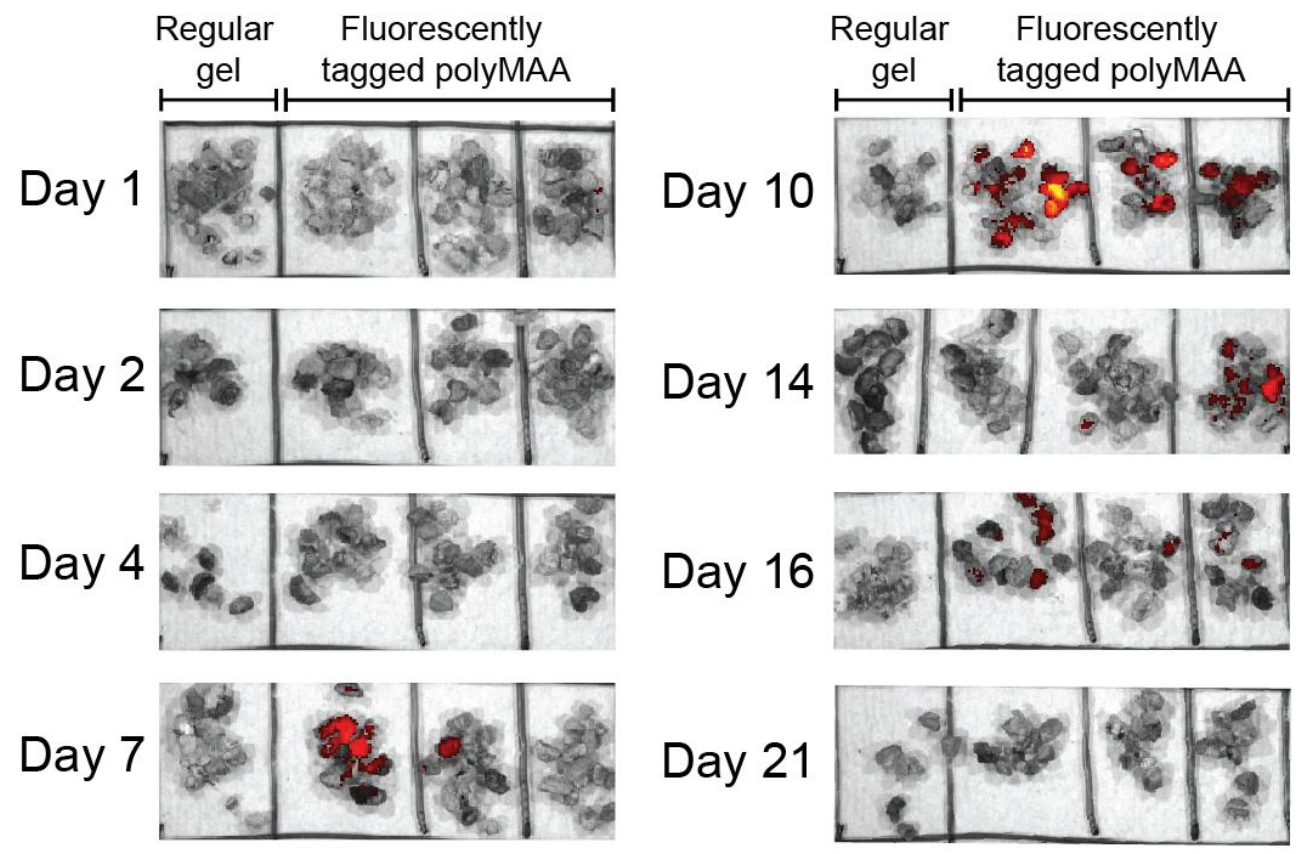

Figure S7: PolyMAA was found in the urine. Bedding of mice receiving the non-fluorescent $(\mathrm{n}=1)$ or fluorescent $(\mathrm{n}=3)$ polyMAA-collagen gel. Fluorescence was detected on the bedding from day 7 until day 14 post-wounding. The peak of fluorescence was obtained at day 10 . 